\title{
Calculating the area of the Trapezium by Using the Length of the Non Parallel Sides: A New Formula for Calculating the area of Trapezium
}

\author{
Salman Mahmud* \\ Student of BIAM Model School and College Bogura, Bangladesh
}

*Corresponding Author: Salman Mahmud, Student of BIAM Model School and College Bogura, Bangladesh

\begin{abstract}
There are some ways to calculate the area of the trapezium. We can easily calculate the area of the trapezium by using the length of the parallel sides and the vertical distance between them. We can also use Brahmagupta's formula and Heron's formula. But in this article I will use the length of one non parallel side and the vertical distance between this non parallel side (or the increased part of this non parallel side) and the midpoint of the other non-parallel side. For this at first I have to prove a theorem and the theorem is, if we imagine one non parallel side of the trapezium as the base of a triangle and the midpoint of other non-parallel side as the vertex of that triangle, the area of the triangle will be half of the area of the trapezium. After proving this theorem, we will easily get a new formula to calculate the area of the trapezium.
\end{abstract}

Keywords: non-parallel, vertex, vertical, increased part.

\section{INTRODUCTION}

A trapezium, also known as a trapezoid, is a quadrilateral in which a pair of sides are parallel, but the other pair of opposite sides are non-parallel. The term trapezium has been in use in English since 1570, from Late Latin trapezium, from Greek literally "a little table". The first recorded use of the Greek word translated trapezoid was by Marinus Proclus (412 to 485 AD) in his Commentary on the first book of Euclid's Elements. There are some formulas for calculating the area of the trapezium. But in this article a new, short and easy formula has been shown. According to this study, the multiplication of the length of a non-parallel side of a trapezium and the vertical distance between this non-parallel side (or the increased part of this non-parallel side) and the midpoint of other non-parallel side is equal to the area of the trapezium. To prove this formula, at first a theorem has been proven (mentioned in abstract).

\section{The Proof of THE NEW ForMula}

At first I want to mention the formula and the formula is, the multiplication of the length of a nonparallel side of a trapezium and the vertical distance between this non-parallel side (or the increased part of this non-parallel side) and the midpoint of other non-parallel side is equal to the area of the trapezium. I have to prove this. To prove this formula at first I have to prove a theorem which I mentioned before and the theorem is,

if we imagine one non parallel side of the trapezium as the base of a triangle and the midpoint of other non parallel side as the vertex of that triangle, the area of the triangle will be half of the area of the trapezium.

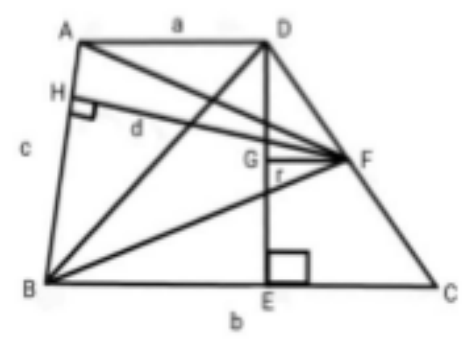


Proof:

Figure 1: Here $\mathrm{ABCD}$ is a trapezium and $\mathrm{AD} \| \mathrm{BC}$.

Here, $\mathrm{AD}\|\mathrm{BC}, \mathrm{AD}=\mathrm{a}, \mathrm{BC}=\mathrm{b}, \mathrm{AB}=\mathrm{c}, \mathrm{DE}=\mathrm{r}, \mathrm{FH}=\mathrm{d}, \mathrm{DF}=\mathrm{FC}, \mathrm{FG}\| \mathrm{BC}, \mathrm{DE} \perp \mathrm{BC}$ and $\mathrm{FH} \perp \mathrm{AB}$.

I have to prove that,

The area of $\triangle \mathrm{ABF}=1 / 2$ (the area of the trapezium $\mathrm{ABCD}$ )

In the case of $\triangle \mathrm{BCD}$,

The base of this triangle $=\mathrm{BC}=\mathrm{b}$

The height of this triangle $=\mathrm{DE}=\mathrm{r}$

So, the area of $\triangle \mathrm{BCD}(=1 / 2 \times$ base $\times$ height $)=1 / 2 \times \mathrm{BC} \times \mathrm{DE}$

$$
=1 / 2 \times b r
$$

Again, in the case of $\triangle \mathrm{ABD}$,

$$
\begin{aligned}
& \text { Base }=\mathrm{AD}=\mathrm{a} \\
& \text { Height }=\mathrm{DE}=\mathrm{r}
\end{aligned}
$$

So, the area of $\triangle \mathrm{ABD}=1 / 2 \times \mathrm{ar}$

Now,

Area of trapezium $\mathrm{ABCD}=$ area of $\triangle \mathrm{BCD}+$ area of $\triangle \mathrm{ABD}$

$$
\begin{aligned}
& =1 / 2 \times b r+1 / 2 \times a r \\
& =1 / 2 \times(b+a) \times r \\
& =1 / 2(a+b) r
\end{aligned}
$$

Here, $\triangle \mathrm{DEC}$ is a right triangle. So, $\mathrm{CD}$ is the hypotenuse and DE, EC are the legs.

As we can see, $\mathrm{F}$ is the midpoint of $\mathrm{CD}$. So, $\mathrm{CF}=\mathrm{FD}$ and we know FG $\| \mathrm{BC}$.

That's why $\mathrm{G}$ is the midpoint of DE.

So, $\mathrm{DG}=\mathrm{GE}=\mathrm{r} / 2$

In the case of $\triangle \mathrm{ADF}$,

$$
\begin{aligned}
& \text { Base }=\mathrm{AD}=\mathrm{a} \\
& \text { Height }=\mathrm{DG}=\mathrm{r} / 2
\end{aligned}
$$

So, the area of $\triangle \mathrm{ADF}=1 / 2 \times \mathrm{a}(\mathrm{r} / 2)$

$$
=\operatorname{ar} / 4
$$

Again, in the case of $\triangle \mathrm{BCF}$,

$$
\begin{aligned}
& \text { Base }=B C=b \\
& \text { Height }=E G=r / 2
\end{aligned}
$$

So, the area of $\triangle \mathrm{BCF}=1 / 2 \times \mathrm{b}(\mathrm{r} / 2)$

$$
=\mathrm{br} / 4
$$

Now,

Area of $\triangle \mathrm{ABF}=$ area of trapezium $\mathrm{ABCD}-($ area of $\triangle \mathrm{ADF}$ + area of $\triangle \mathrm{BCF}$ )

Now,

$$
\begin{aligned}
& =1 / 2(a+b) r-a r / 4-b r / 4 \\
& =1 / 4(a+b) r
\end{aligned}
$$

Area of $\triangle \mathrm{ABF} /$ area of the trapezium $\mathrm{ABCD}$

$$
=\{1 / 4(a+b) r\} /\{1 / 2(a+b) r\}
$$

Or, area of $\triangle \mathrm{ABF} /$ area of the trapezium $\mathrm{ABCD}=1 / 2$

So, area of $\triangle \mathrm{ABF}=1 / 2($ area of the trapezium $\mathrm{ABCD})$

(Proved) 
In the case of $\triangle \mathrm{ABF}$,

$$
\begin{aligned}
\text { Base }=A B=c & \\
\text { Height }=H F=d & {[\text { Because } H F=d \text {, is the }} \\
& \text { vertical distance between } \\
& A B \text { and the point } F \text { (vertex } \\
& \text { of } \triangle A B F)]
\end{aligned}
$$

So, the area of $\triangle \mathrm{ABF}=1 / 2 \times \mathrm{AB} \times \mathrm{HF}$

$$
=1 / 2 \times \mathrm{cd}
$$

By using the above mentioned theorem we can write,

$$
1 / 2 \times c d=1 / 2(\text { area of the trapezium } \mathrm{ABCD})
$$

Or, area of the trapezium $A B C D=2 \times(1 / 2 \times c d)$

So, area of the trapezium $\mathrm{ABCD}=\mathrm{cd}$

Here, $c$ is the length of a non-parallel side $(\mathrm{AB})$ of the trapezium $\mathrm{ABCD}$ and $\mathrm{d}$ is the vertical distance between this non-parallel side $(\mathrm{AB})$ and the midpoint of other non-parallel side $\mathrm{CD}$.

So, the multiplication of the length of a non-parallel side of a trapezium and the vertical distance between this non-parallel side (or the increased part of this non-parallel side) and the midpoint of other non-parallel side is equal to the area of the trapezium.

(Proved)

\section{CONCLUSION}

In this article, I have given a new formula to calculate the area of the trapezium. The conclusions of this study are summarized as follows:

1.By using the above mentioned formula we can easily calculate the area of the trapezium and it is very easy and short formula than the other formulas. Because in this case we need only two information about the trapezium.

2. We will be able to know more information about the trapezium.

\section{ACKNOWLEDGEMENTS}

I should Thanks MD. Shah Alam and MST. Sabina Yesmin for providing me with the help materials.

\section{REFERENCES}

[1] S. Alam, helped in the theory, Teacher at Bihar M.A.M High School, Bogura, Bangladesh.

\section{AUTHOR'S BIOGRAPHY}

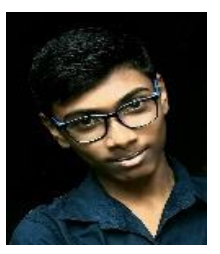

Salman Mahmud, is still a student of BIAM Model school and College, Bogura, Bangladesh. His core research interest is in modern physics. But he is interested in all the sectors of science

Citation: Salman Mahmud, (2019). Calculating the area of the Trapezium by Using the Length of the Non Parallel Sides: A New Formula for Calculating the area of Trapezium. International Journal of Scientific and Innovative Mathematical Research (IJSIMR), 7(4), pp.25-27. http://dx.doi.org/10.20431/2347-3142.0704004

Copyright: ( $) 2019$ Authors, this is an open-access article distributed under the terms of the Creative Commons Attribution License, which permits unrestricted use, distribution, and reproduction in any medium, provided the original author and source are credited. 